\title{
Frailty and Sarcopenia
}

\author{
Finbarr C. Martin and Anette Hylen Ranhoff
}

\subsection{Frailty}

Frailty is generally understood as a progressive age-related decline in physiological systems that results in decreased reserves, which confers extreme vulnerability to stressors and increases the risk of a range of adverse health outcomes. There are however two distinct concepts that emerge from the clinical and research literature. The first is of a syndrome associated with underlying physiological and metabolic changes that are responsible for driving progressive physical and cognitive impairments through to loss of functional capacity, often helped on the way by acute or chronic disease or injury. This can be encapsulated by the definition proposed some two decades ago and still valid [1]. As a result, the frail person is at increased risk of disability or death from minor external stresses.

The second concept underpins a pragmatic approach, which treats frailty as a collection of risk factors for future adverse events, whilst not necessarily bearing a direct pathophysiological relationship to these outcomes. As discussed later these positions are not incompatible. Either way, both epidemiologically and

This chapter is a component of Part 1: Background.

For an explanation of the grouping of chapters in this book, please see Chapter 1: 'The Multidisciplinary Approach to Fragility Fractures Around the World-An Overview'.

\section{F. C. Martin ( $ه)$}

Population Health Sciences, King's College London, London, UK

e-mail: finbarr.martin@kcl.ac.uk
A. H. Ranhoff
Department of Clinical Science, University of Bergen, Bergen, Norway
Department of Medicine, Diakonhjemmet Hospital, Oslo, Norway
Norwegian Institute of Public Health, Oslo, Norway
e-mail: anette.ranhoff@uib.no 
conceptually, frailty overlaps with but is distinct from multimorbidity and disability [2]. In cross-sectional studies, some frail individuals are neither multimorbid nor disabled, but multimorbid individuals are more likely than others to be frail, and frail individuals are by definition more likely to develop a new disability. Frailty could therefore be looked upon as a risk factor for functional decline, in line with more traditional risk factors such as hypertension, hypercholesterolemia, obesity and smoking [3].

\subsubsection{The Nature of Frailty}

There are several diagnostic definitions and measures of frailty, validated in various populations in terms of predicting an increased incidence of adverse outcomes such as new disability, hospitalisation and death. The two best-established approaches are the phenotype model developed by Fried's group in the United States [4] and the deficit accumulation model developed by Rockwood and Mitnitski in Canada [5]. Both have been validated subsequently in unrelated cohorts internationally.

The phenotype approach was generated empirically from a larger number of candidate features tested in a longitudinal dataset, and analysis resolved them into five components - unintentional weight loss, self-reported fatigue, low physical activity and impairment of grip strength and gait speed comparative to relevant population norms. Three or more abnormalities define frailty, with pre-frailty defined as the presence of one or two. The criteria for judging abnormality is illustrated in Fig. 4.1, but in practice, subsequent researchers have adapted criterion definitions to the data available.

\section{The Fried Phenotype Model of Frailty}

\section{Weight loss}

Exhaustion

Low energy expenditure

Slow gait speed

Weak grip strength
Self-reported weight loss of more than $4.5 \mathrm{~kg}$ or recorded weight loss of " $5 \%$ per year

Self-reported exhaustion on US Center for Epidemiological Studies depression scale73

(3-4 days per week or most of the time)

Energy expenditure $<383 \mathrm{kcal} /$ week (men) or $<270 \mathrm{kcal} /$ week (women)

Standardised cut-off times to walk $4.57 \mathrm{~m}$, stratified by sex and height

Grip strength, stratified by sex and body-mass index

Fig. 4.1 The frailty phenotype 
This phenotype model, therefore, does not explicitly include cognitive or psychosocial features that are also well known to be predictive of adverse health outcomes, but these domains may impact any of the five dimensions, for example, a low mood may be associated with self-assessed fatigue, and cognitive impairment is associated with slower gait. Nevertheless, there is substantial evidence that this predominantly physical frailty phenotype has predictive power for adverse health outcomes in several cohorts of older people.

The deficit accumulation approach is quite different. It operationalises frailty as the sum total of age-related factors that may be regarded as detrimental ("deficits"). These could be symptoms, sensory impairments, abnormal clinical findings or laboratory test results, diseases, disabilities or lack of social support. Suitable parameters are those that are increasingly prevalent with advancing age but do not saturate and are credibly associated with health. Thus, grey hair is not suitable! Generally, each is regarded as present or absent and thus accorded a score of 0 or 1 , although some variables lend themselves to be divided in three or occasionally more grades, so become fractions of one. The total score, termed the frailty index (FI), is calculated from the sum of all the deficit scores divided by the number of items included. The theoretical range of the FI is therefore between 0 (no deficits apparent, good health) to 1 (deficits in every item), but in practice, a number of studies have now shown that survival is rare with scores above about 0.7 . The deficit accumulation model is an approach rather than a fixed tool and is therefore highly flexible. A FI can be constructed from suitable variables in any comprehensive dataset about an individual as long as it covers a broad range of these health-related domains and includes upwards of 30 items. Despite these two approaches to diagnosing frailty being quite distinct, they perform fairly similarly in identifying frailty when applied to a common dataset $[6,7]$.

\subsubsection{Epidemiology of Frailty}

Whatever approach is used to define frailty, it becomes more prevalent with increasing age. The prevalence in community versus institutional care settings is $12 \%$ (95\% CI 10-15\%) and 45\% (95\% CI 27-63\%), respectively. When using broader definitions than the physical phenotype, the prevalence increases to $16 \%$ (95\% CI $7-29 \%$ ) [8]. Frailty prevalence rises to $20-50 \%$ by age $85+$ [9] and is more common in women, but several studies suggest that women are more resilient to frailty than men. Geographical differences in frailty prevalence may be related to health inequalities, as rates are significantly associated with national economic indicators. Differences within countries may also be associated with socioeconomic factors including social deprivation [10]. Frailty is a dynamic syndrome and may be reversible-people move in and out of a frailty state [11]. However, there are few studies of frailty trajectories. 


\subsubsection{How Does Frailty Develop?}

Frailty may be best understood from the standpoint of ageing and evolution. A universal result of living is the gradual and progressive process of acquiring deleterious changes to body structure and function, affecting all individuals to variable degrees and not associated with a specific external cause. These ageing-related impairments result from the lifelong accumulation of unrepaired molecular and cellular damage, which take multiple forms and impact cell survival, protein synthesis and the efficiency of damage detection and repair processes. The pathophysiological pathways that result from this damage are not fully elucidated, but candidates include cytokines and other components of the inflammatory response [12,13]. The defence and repair mechanisms are generally good enough in earlier life to enable normal growth, development and reproduction, but did not evolve to provide indefinite protection in older age. There was no evolutionary pressure to do so and since all metabolic processes use energy (ultimately from food), it makes biological sense to develop enough but not surplus repair capacity. These age-related changes are accompanied by an increased chance of certain "degenerative" diseases, but these are not universal. Disability results from the critical impairment of specific attributes, such as strength or balance, these impairments arising from ageing or disease or more usually both.

\subsubsection{Assessment of Frailty in Clinical Practice}

The scope and detail of assessment needed and the choice of assessment tool should be tailored to the population being assessed and the purpose of the assessment. For example, many of the functional tests such as walking speed and the Timed Up and Go test are not feasible in patients with acute hip fractures. Neither the phenotype model nor the FI is particularly feasible in routine clinical practice, so simpler tools are more commonly used such as the Clinical Frailty Scale [14] or the Edmonton Frail Scale [15]. The Clinical Frailty Scale uses descriptors covering the domains of mobility, energy, physical activity, and function to enable a standard clinical assessment to characterise nine levels from very fit, healthy through very severely frail to terminally ill. (Fig. 4.2). This provides a feasible description based on routine clinical assessment but does not conceptually distinguish frailty from multimorbidity or disability. Its mortality prediction is comparable to that of the more detailed FI and it is useful in settings where a quick impression can help indicate what clinical decisions need to be considered.

The Edmonton scale requires a number of specific but fairly simple clinical measures to be performed, which would be additional to routine clinical practice. The domains included are cognition (the clock drawing test), general health status, functional ability, social support, medication use, nutrition, mood, continence and a mobility function test- the Timed Up and Go. Scores range from 0 to 17, scores of 8 or above usually being considered to be frail, but relevant cut-offs can be established empirically depending upon the purpose. For example, the prediction of 


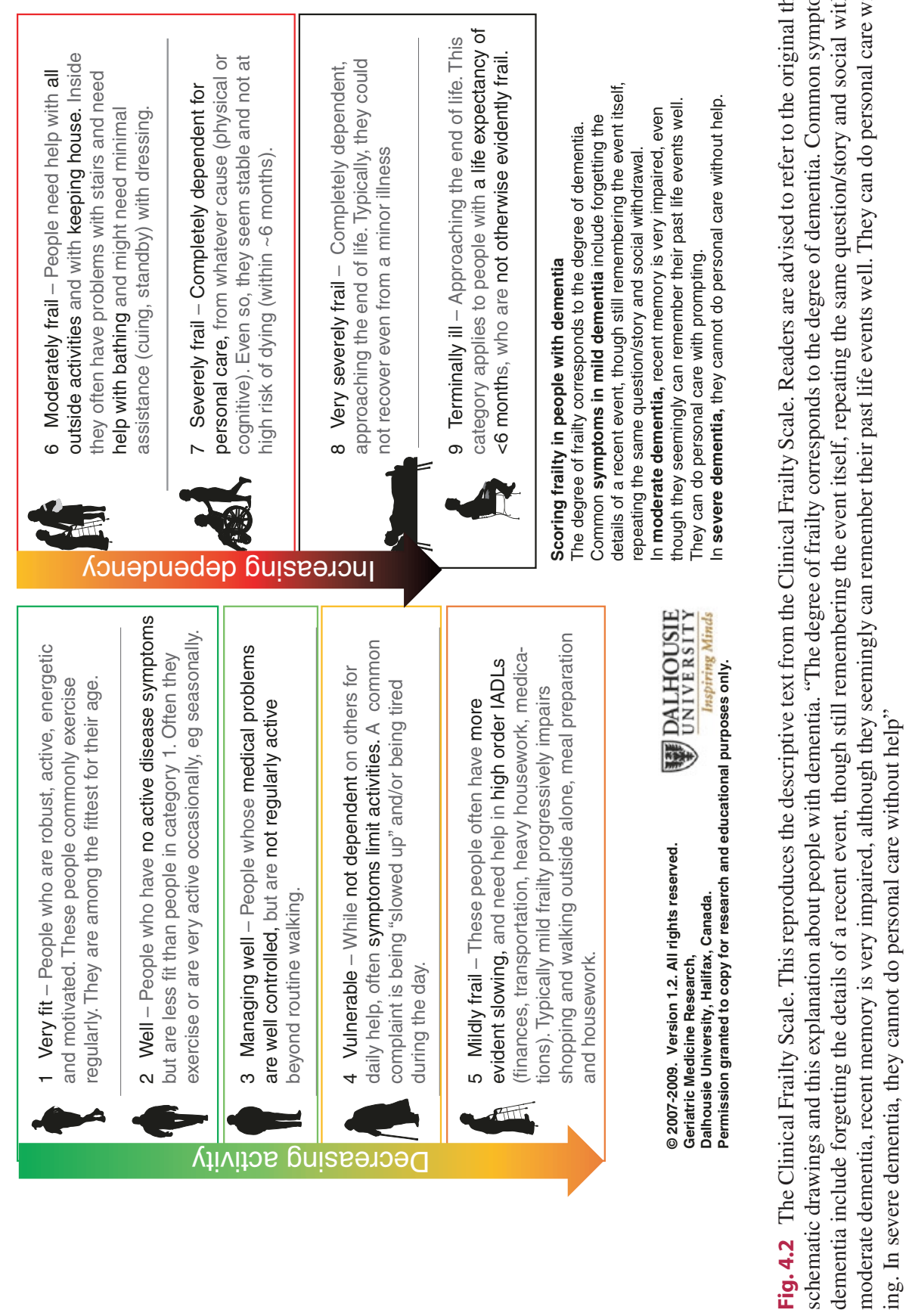

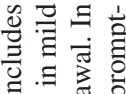

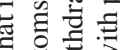
쿄 3 드음 き 잉 ن. 용 ปี

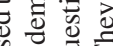
O on 屯ै 용 i 음 ปั 층 现 त् 讷 \&. ह 음 $\Xi \Xi$ .

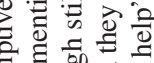
원 돌 3 击 3

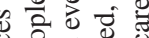
范 言要自 एक त्र

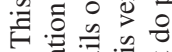

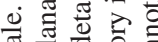

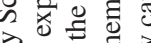
틀.

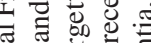

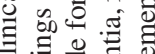

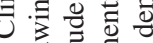

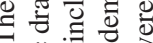
N 氙苋

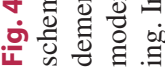




\begin{tabular}{|c|c|c|}
\hline 1 & Are you more than 85 years? & Yes $=1$ point \\
\hline 2 & Male? & Yes $=1$ point \\
\hline 3 & In general, do you have any health problems that require you to limit your activities? & Yes $=1$ point \\
\hline 4 & Do you need someone to help you on a regular basis? & Yes $=1$ point \\
\hline 5 & In general, do you have any health problems that require you to stay at home? & Yes $=1$ point \\
\hline 6 & In case of need, can you count on someone close to you? & No= 1 point \\
\hline \multirow[t]{2}{*}{7} & Do you regularly use a stick, walker or wheelchair to get about? & Yes $=1$ point \\
\hline & Total & \\
\hline
\end{tabular}

Fig. 4.3 The PRISMA score

likely higher rates of postoperative complications may be associated with lower scores. In contrast to some other tools, the Edmonton scale identifies potential targets for intervention across a number of clinically important domains.

In community or primary care settings, the issue may be to identify a target group for health-promoting interventions such as optimising nutrition and increasing physical activity levels. Here a more simple screening approach may be needed. A recent systematic review assessing available tools suggested that PRISMA-7 may be the most accurate [16], a score of 3 or more suggesting the increased likelihood of incident disability [17] (Fig. 4.3).

\subsubsection{Incorporating Frailty into Treatment Plans and Service Design}

In general, there is not sufficient evidence for screening programmes for frailty [18]. Case finding in clinical settings could be carried out in two phases, using a short screening test and then confirming the diagnosis using a comprehensive geriatric assessment (CGA) if a geriatric service is available, or at least an assessment of nutrition and muscle function. The need then is to provide intervention or package of interventions for those deemed to be pre-frail or frail to prevent, slow or reverse frailty [19]. Patients with fragility fractures could be assessed for frailty in the acute setting by using, for example, the Clinical Frailty Scale based on information from the patient and next-of-kin about the pre-fracture status. In a rehabilitation phase, the Edmonton scale or á full CGA would enable an individually tailored programme to be applied.

\subsection{Sarcopenia}

Sarcopenia was the term suggested by Rosenberg for the well-recognised loss of muscle with ageing [20]. It is a major component of frailty. The diagnosis, treatment and prevention of sarcopenia is recommended to become part of routine 
clinical practice [21]. Skeletal muscle accounts for a third or more of total body mass. As well as movement, muscle plays a key role in temperature regulation and metabolism. Low muscle mass is associated with poor outcomes from acute illness, probably because of the reduced metabolic reserve, as muscle is a reservoir for proteins and energy that can be used for the synthesis of antibodies and gluconeogenesis.

\subsubsection{The Nature of Sarcopenia}

Sarcopenia is characterised by motor neurone loss, reduced muscle mass per motor unit, relatively more loss of fast-twitch fibres and reduced strength per unit of crosssectional area. Muscle fibres are lost by drop-out of motor neurones. Reinnervation of fibres by sprouting from surviving neurones cause less even distribution of fibre types cross-sectionally and a relatively greater loss of type II fibres that are associated with the generation of power (the product of force generation and speed of muscle contraction) [22].

Muscle mass and strength are of course related but not linearly [23]. Function is more important than mass for physical performance and disability [24] Leg power accounts for $40 \%$ of the decline in functional status with ageing [25]. Men who maintain physical activity into their 80 s show compensatory hypertrophy of muscle fibres to compensate for the decrease in fibre number. Loss of efficiency also results from an accumulation of fat within and between fibres and an increase in non-contractile connective tissue material. Muscle strength and function also depend on neuromuscular integrity and muscle performance as well as muscle characteristics. Indeed, the force produced by external electrical stimulation to large muscle groups such as quadriceps exceeds that which can be achieved by maximal voluntary contraction, emphasising the importance of non-muscle factors.

\subsubsection{Epidemiology}

A systematic review on the prevalence of sarcopenia by the European Working Group on Sarcopenia in Older People (EWGSOP) criteria reported a variable prevalence from 1 to $29 \%$ in persons living in the community, 14-33\% in those living in long-term care institutions and $10 \%$ for those in acute hospital care [26]. A higher prevalence at $30 \%$ was reported from a Norwegian population of hospitalised older persons [27]. In most of these studies, the prevalence of sarcopenia increased with age, but the effect of sex varied. A study from Iceland found an increase in the prevalence of sarcopenia from 7 to $17 \%$ from age 75 to 80 . In older (65+) hip fracture patients sarcopenia is found in $17-74 \%$, the highest prevalence in Chinese male patients. In a selected population of previously home-dwelling older hip fracture patients, mean age 79 years old, $38 \%$ had sarcopenia according to the EWGSOP 2010 definition [28]. 


\subsubsection{How Does Sarcopenia Develop?}

Muscle fibre development occurs before birth but fibres enlarge during childhood reaching a peak in early adulthood. Mass and function then gradually decline into older age [29]. Peak mass is affected by intra-uterine, genetic and early life influences. The decline is affected by physical activity, nutrition and sex. It is more pronounced in women from menopause onwards. Adding to the inevitable moderate decline of some $15-25 \%$ by old age is the impact of acute illness or chronic conditions, which have generally negative effects through the mechanisms of catabolic stress, reduced food intake and physical inactivity. The loss of muscle mass is thought to be multifactorial with potential factors illustrated in Fig. 4.4.

The factors implicated in sarcopenia overlap with those for frailty. A central feature of sarcopenia is a decrease in the rate of muscle protein synthesis. This leads to reduced protein levels including mitochondrial oxidative enzymes responsible for enabling work intensity. The age-related shift of the hormonal balance towards low testosterone, growth hormone and IGF-I contributes to the lower muscle protein synthesis rates, which also limits the structural recovery from muscle damage or apoptosis and possibly reduces the synthetic stimulus of exercise [30]. The role of cytokines such as interleukins IL- $1 \beta$ and IL- 6 and TNF- $\alpha$ is less certain. They play

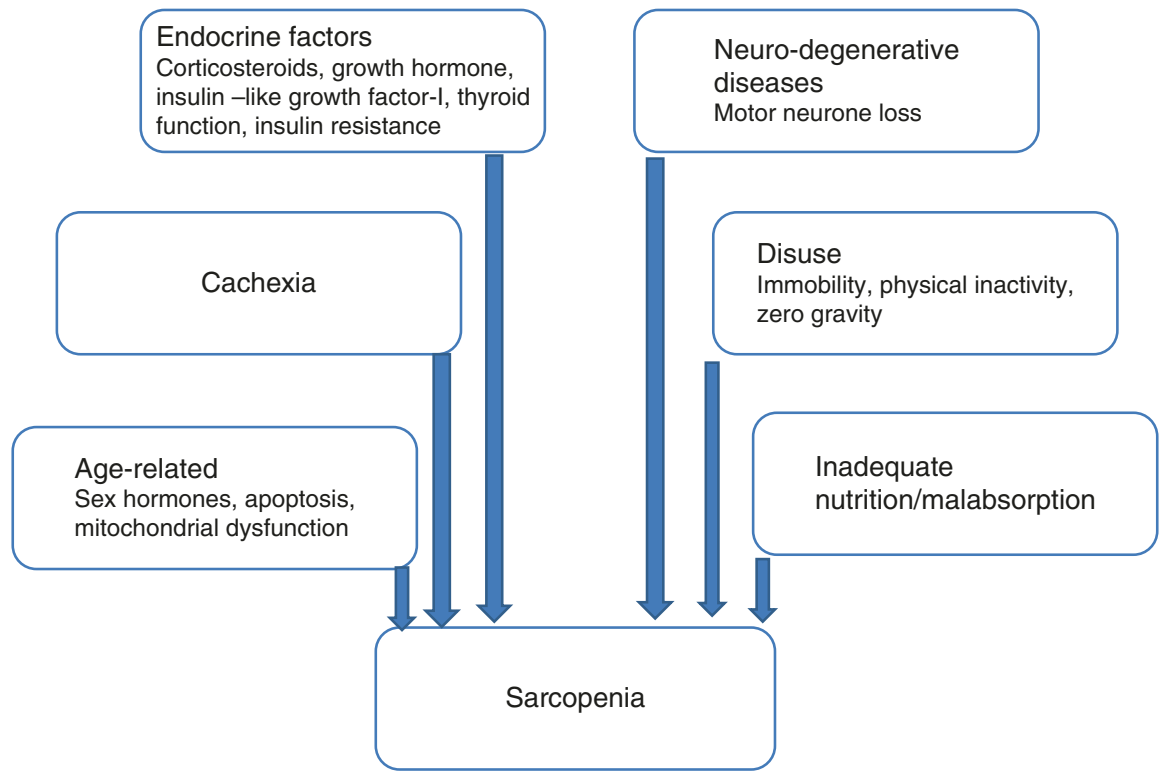

Fig. 4.4 Aetiological factors and mechanisms of sarcopenia (Adapted from Cruz-Jentoft AJ, Baeyens JP, Bauer JM, Boirie Y, Cederholm T, Landi F, Martin FC, Michel JP, Rolland Y, Schneider SM, Topinková E, Vandewoude M, Zamboni M. Sarcopenia: European consensus on definition and diagnosis: Report of the European Working Group on Sarcopenia in Older People. Age Ageing. $2010 \mathrm{Jul} ; 39(4): 412-23)$ 
a role in the catabolic processes of acute illness and chronic inflammatory conditions, but whether the small differences in circulating levels associated with frailty reported from some population studies are relevant to the age-related sarcopenia is not established.

\subsubsection{Assessing Sarcopenia in Clinical Practice}

There are several different diagnostic definitions resulting in the range of prevalence rates reported earlier from community-dwelling populations of older people. A consensus definition and approach to screening and classification has been proposed by EWGSOP established by the European Geriatric Medicine Society, first in 2010, and with a revised version in 2018 [31]. This is shown in Fig. 4.5. Measuring gait speed of ambulant people is feasible in almost any community setting and is a useful global indicator of health, slower gait being associated with a greater likelihood of incident disability, falls, institutionalisation and death [32]. Grip strength was

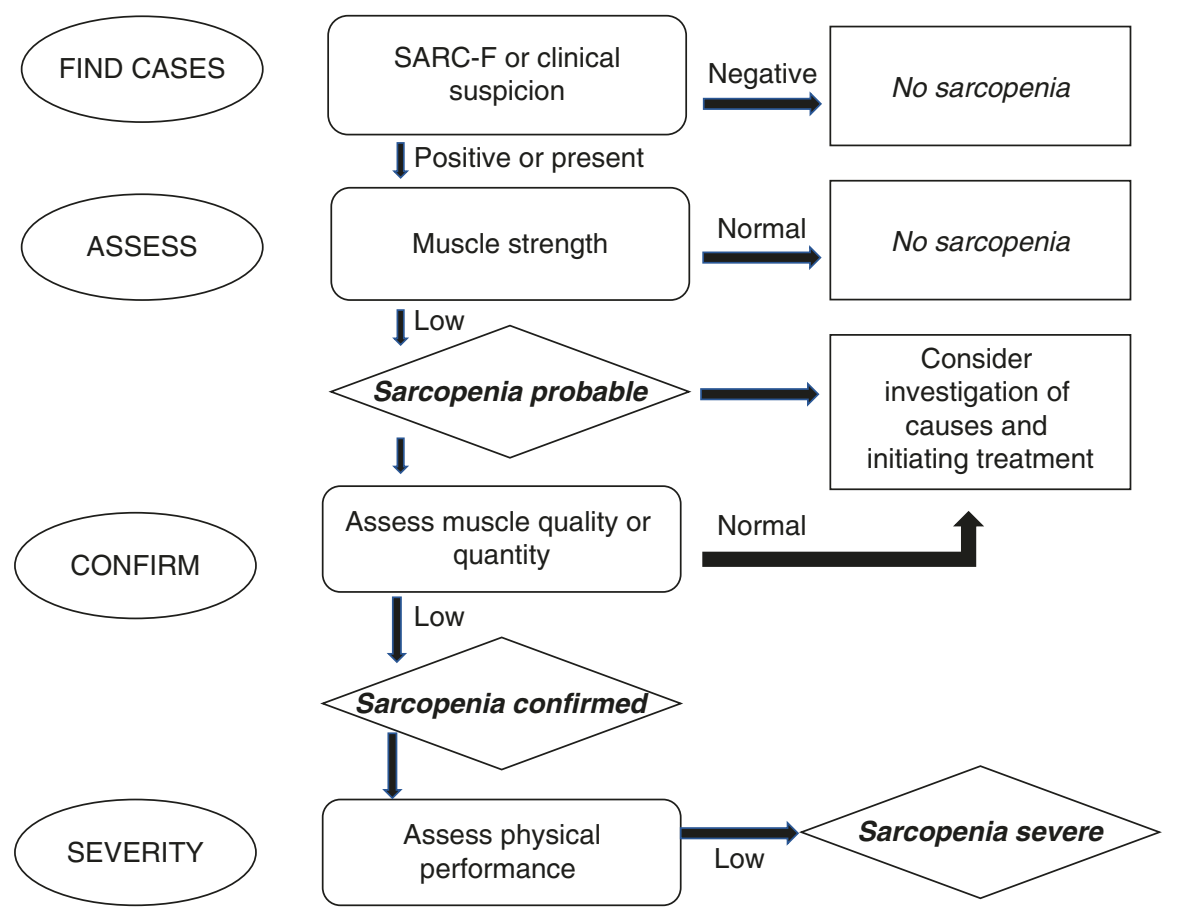

Fig. 4.5 The EWGSOP algorithm for the diagnosis and grading of sarcopenia (Adapted from Cruz-Jentoft AJ, Bahat G, Bauer JM, et al. (2019) Sarcopenia: revised European consensus on definition and diagnosis: Age Ageing; 48(1):16-31). Further details on how to apply this algorithm can be found in this paper, with suggestions for feasible and reliable methods to assess muscle strength, quality and quantity, and physical performance 
chosen as it is a portable, simple, reliable and valid proxy measure of body strength, and has a good correlation with lower limb physical performance. Low grip strength of community-dwelling older people is associated with falls, increased incident disability and earlier mortality. It also predicts slower and less complete functional recovery from illness in men [33].

Measurement of muscle mass can be done with CT scan or, less accurately, with impedance techniques or anthropometry (measurements of upper mid-arm circumference, estimation of upper mid-arm muscle cross-sectional area, measurement of mid-calf circumference) [31]. Ultrasound is a promising emerging technique but not yet in routine use. Assessment of sarcopenia with the above-mentioned methods may not be feasible in clinical populations such as after lower limb fractures, and measurement of muscle function is more important than measurement of muscle mass in clinical practice. An indication of pre-fracture severe sarcopenia can be obtained, using the Sarc-F tool, which is a brief questionnaire about muscle function (mobility and ability to carry a certain weight). The Sarc-F tool is available in many languages [34].

\subsubsection{Incorporating Sarcopenia into Treatment Plans and Service Design}

Sarcopenia has recently been recognized as a clinical diagnosis with a corresponding code in the ICD system. For the diagnosis of sarcopenia, either the definitions from EWGSOP or the Foundation for the National Institutes of Health (FNIH) are recommended. But for rapid screening and special patient groups, such as hip fracture patients, rapid screening with the SARC-F is recommended. All health personnel working with older patients should be aware of sarcopenia.

\subsection{The Implications of Frailty and Sarcopenia on Falls, Fractures and the Recovery After Fractures}

With the exception of the vertebra, most fractures in older people are related to falls. Poor bone health makes a fracture more likely, but from the population perspective, risk of falling is more predictive of fractures than bone mineral density [35], leading to the suggestion that the focus of primary fracture prevention must rest with identifying those at risk of falls rather than those with osteoporosis [36]. After a fragility fracture, however, it is imperative to consider falls prevention and bone health as described in detail in Chapters 14-16 of the IIIrd Pillar.

There are common risk factors and overlap in the biology of frailty, sarcopenia and osteoporosis. Frailty predicts lower bone mineral density, an increased likelihood of falls, vertebral and hip fractures [37, 38]. Sarcopenia and osteoporosis often co-exist and shared risk factors have given rise to the notion of osteosarcopenioa [39]. The typical hip fracture sufferer is a frail woman more than 80 years, so prevention requires a multicomponent approach embracing frailty. 
For older people with osteoporosis, a consensus panel recommended a multicomponent exercise programme including resistance and balance training [40]. In general, physical exercise programmes are shown to be effective for reducing or postponing frailty with benefits more likely if conducted in groups. Physical exercise with nutritional supplementation, supplementation alone, cognitive training and combined treatment do also show a favourable effect on frailty outcomes [41]. Recognition of frailty is also key in the management of those who have fractured. For example, frailty, as assessed with the FI, was associated with longer hospital length of stay and reduced chance of returning home within 30 days after hip fracture [42].

\subsection{Concluding Statement}

There are close links epidemiologically, biologically and clinically between frailty, sarcopenia, poor bone health and the geriatric syndrome of falls. Older people who have had a fall and/or a fracture should be assessed for frailty and sarcopenia to better develop a care plan. This calls for an integrated clinical approach to the prevention and treatment of fragility fractures.

\section{References}

1. Campbell AJ, Buckner DM (1997) Unstable disability and the fluctuations of frailty. Age Ageing 26(4):315-318

2. Fried LP, Ferrucci L, Darer J, Williamson JD, Anderson G (2004) Untangling the concepts of disability, frailty, and comorbidity: implications for improved targeting and care. J Gerontol Med Sci 59(3):255-263

3. Cesari M, Pérez-Zepeda MU, Marzetti E (2017) Frailty and multimorbidity: different ways of thinking about geriatrics. J Am Med Dir Assoc 18(4):361-364

4. Fried LP, Tangen CM, Walston J et al (2001) Frailty in older adults: evidence for a phenotype. J Gerontol A Biol Sci Med Sci 56:M146-M156

5. Rockwood K, Song X, MacKnight C, Bergman H, Hogan DB, McDowell I, Mitnitski A (2005) A global clinical measure of fi tness and frailty in elderly people. Can Med Assoc J 173(5):489-495

6. Romero-Ortuno R (2013) The frailty instrument for primary care of the Survey of Health, Ageing and Retirement in Europe predicts mortality similarly to a frailty index based on comprehensive geriatric assessment. Geriatr Gerontol Int 13:497-504

7. Rockwood K, Andrew M, Mitnitski A (2007) A comparison of two approaches to measuring frailty in elderly people. J Gerontol A Biol Sci Med Sci 62(7):738-743

8. O'Caoimh R, Galluzzo L, Rodríguez-Laso Á, Work Package 5 of the Joint Action ADVANTAGE et al (2018) Prevalence of frailty at population level in European ADVANTAGE Joint Action Member States: a systematic review and meta-analysis. Ann Ist Super Sanita 54(3):226-238

9. Collard RM, Boter H, Schoevers RA, Oude Voshaar RC (2012) Prevalence of frailty in community- dwelling older persons: a systematic review. J Am Geriatr Soc 60(8):1487-1492

10. Lang IA, Hubbard RE, Andrew MK, Llewellyn DJ, Melzer D, Rockwood K (2009) Neighborhood deprivation, individual socioeconomic status, and frailty in older adults. J Am Geriatr Soc 57(10): 1776-1780 
11. Xue QL, Bandeen-Roche K, Varadhan R, Zhou J, Fried LP (2008) Initial manifestations of frailty criteria and the development of frailty phenotype in the Women's Health and Aging Study II. J Gerontol A Biol Sci Med Sci 63(9):984-990

12. Clegg A, Young J, Iliffe S, Rikkert MO, Rockwood K (2013) Frailty in elderly people. Lancet 381(9868):752-762

13. Puts MTE, Visser M, Twisk JWR, Deeg DJH, Lips P (2005) Endocrine and inflammatory markers as predictors of frailty. Clin Endocrinol 63:403-411

14. National Institute for Health and Care Excellence (2016) Multimorbidity: assessment, prioritisation and management of care for people with commonly occurring multimorbidity. NICE guideline. http://www.nice.org.uk. Accessed 12 Sept 2019

15. Rolfson DB, Majumdar SR, Tsuyuki RT et al (2006) Validity and reliability of the Edmonton Frail Scale. Age Ageing 35:526-529

16. Hoogendijk EO, Van Der Horst HE, Deeg DJH et al (2013) The identification of frail older adults in primary care: comparing the accuracy of five simple instruments. Age Ageing 42:262-265

17. Raiche M, Hebert R, Dubois MF (2008) PRISMA-7: a case-finding tool to identify older adults with moderate to severe disabilities. Arch Gerontol Geriatr 47:9-18

18. Dent E, Martin FC, Bergman H, Woo J, Romero-Ortuno R, Walston JD (2019) Management of frailty: opportunities, challenges, and future directions. Lancet 394:1376-1386

19. Rodríguez-Laso Á, O’Caoimh R, Galluzzo L, Carcaillon-Bentata L, Beltzer N, Macijauskiene J, Albaina Bacaicoa O, Ciutan M, Hendry A, López-Samaniego L, Liew A, Work Package 5 of the Joint Action ADVANTAGE (2018) Population screening, monitoring and surveillance for frailty: three systematic reviews and a grey literature review. Ann Ist Super Sanita 54(3):253-262

20. Rosenberg IH (1989) Summary comments. Am J Clin Nutr 50:1231-1233

21. Cruz-Jentoft AJ, Sayer AA (2019) Sarcopenia. Lancet 393(10191):2636-2646

22. Lexell J (1995) Human aging, muscle mass, and fiber type composition. J Gerontol A Biol Sci Med Sci 50:A11-A16

23. Goodpaster BH, Park SW, Harris TB et al (2006) The loss of skeletal muscle strength, mass and quality in older adults. J Gerontol A Biol Sci Med Sci 61:1059-1064

24. Metter EJ, Talbot LA, Schrager M, Conwit R (2002) Skeletal muscle strength as a predictor of all-cause mortality in healthy men. J Gerontol A Biol Sci Med Sci 57(10):B35

25. Foldvari M, Clark M, Laviolette LC et al (2000) Association of muscle power with functional status in community-dwelling elderly women. J Gerontol A Biol Sci Med Sci 55A:M192-M199

26. Cruz-Jentoft AJ, Landi F, Schneider SM, Zuniga C, Arai H, Boirie Y et al (2014) Prevalence of and interventions for sarcopenia in ageing adults: a systematic review. Report of the International Sarcopenia Initiative (EWGSOP and IWGS). Age Ageing 43(6):748-759

27. Jacobsen EL, Brovold T, Bergland A, Bye A (2016) Prevalence of factors associated with malnutrition among acute geriatric patients in Norway: a cross-sectional study. BMJ Open 6(9):e011512

28. Steihaug OM, Gjesdal CG, Bogen B, Kristoffersen MH, Lien G, Hufthammer KO et al (2018) Does sarcopenia predict change in mobility after hip fracture? a multicenter observational study with one-year follow-up. BMC Geriatr 18(1):65

29. Janssen I, Heymsfield SB, Wang ZM, Ross R (2000) Skeletal muscle mass and distribution in 468 men and women aged 18-88 yr. J Appl Physiol 89(1):81-88

30. Giannoulis MG, Martin FC, Nair KS, Umpleby AM, Sonksen P (2012) Hormone replacement therapy and physical function in healthy older men. Time to talk hormones? Endocr Rev 33(3):314-377. https://doi.org/10.1210/er.2012-1002

31. Cruz-Jentoft AJ, Bahat G, Bauer J et al (2019) Sarcopenia: revised European consensus on definition and diagnosis. Age Ageing 48(1):16-31. Erratum in: Age Ageing. 48(4):601, 2019

32. Vermeulen J, Neyens JC, van Rossum E, Spreeuwenberg MD, de Witte LP (2011) Predicting ADL disability in community-dwelling elderly people using physical frailty indicators: a systematic review. BMC Geriatr 11:33 
33. Roberts HC, Syddall HE, Cooper C, Aihie Sayer A (2012) Is grip strength associated with length of stay in hospitalised older patients admitted for rehabilitation? Findings from the Southampton grip strength study. Age Ageing 41(5):641-646

34. Bahat G, Yilmaz O, Oren M et al (2018) Cross-cultural adaptation and validation of the SARC-F to assess sarcopenia: methodological report from European Geriatric Medicine Society Sarcopenia Special Interest Group. Eur Geriatr Med 9:23-28

35. Kaptoge S, Benevolenskaya LI, Bhalla AK et al (2005) Low BMD is less predictive than reported falls for future limb fractures in women across Europe: results from the European Prospective Osteoporosis Study. Bone 36(3):387-398

36. Järvinen TL, Sievänen H, Khan KM, Heinonen A, Kannus P (2008) Analysis—shifting the focus in fracture prevention from osteoporosis to falls. BMJ 336:124-126

37. Sternberg SA, Levin R, Dkaidek S, Edelman S, Resnick T, Menczel J (2014) Frailty and osteoporosis in older women - a prospective study. Osteoporos Int 25(2):763-768

38. Ensrud KE, Ewing SK, Taylor BC et al (2007) Frailty and the risk of falls, fracture, and mortality in older women: the study of osteoporotic fractures. J Gerontol A Biol Sci Med Sci 62:744-751

39. Hassan EB, Duque G (2017) Osteosarcopenia: a new geriatric syndrome. Aust Fam Physician 46(11):849-853

40. Giangregorio LM, Papaioannou A, MacIntyre NJ (2014) Too fit to fracture: exercise recommendations for individuals with osteoporosis or osteoporotic fracture. Osteoporos Int 25:821-835

41. Apóstolo J, Cooke R, Bobrowicz-Campos E et al (2018) Effectiveness of interventions to prevent pre-frailty and frailty progression in older adults: a systematic review. JBI Database System Rev Implement Rep 16(1):140-232

42. Krishnan M, Beck S, Havelock W, Eeles E, Hubbard RE, Johansen A (2014) Predicting outcome after hip fracture: using a frailty index to integrate comprehensive geriatric assessment results. Age Ageing 43(1):122-126

Open Access This book is licensed under the terms of the Creative Commons AttributionNonCommercial-NoDerivatives 4.0 International License (http://creativecommons.org/licenses/ by-nc-nd/4.0/), which permits any noncommercial use, sharing, distribution and reproduction in any medium or format, as long as you give appropriate credit to the original author(s) and the source, provide a link to the Creative Commons license and indicate if you modified the licensed material. You do not have permission under this license to share adapted material derived from this book or parts of it.

The images or other third party material in this book are included in the book's Creative Commons license, unless indicated otherwise in a credit line to the material. If material is not included in the book's Creative Commons license and your intended use is not permitted by statutory regulation or exceeds the permitted use, you will need to obtain permission directly from the copyright holder.

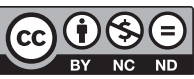

